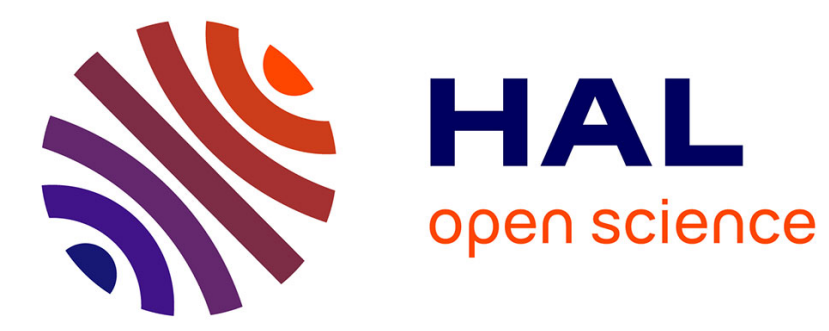

\title{
Models for supercontinuum generation beyond the slowly-varying-envelope approximation
}

Hervé Leblond, Philippe Grelu, Dumitru Mihalache

\section{To cite this version:}

Hervé Leblond, Philippe Grelu, Dumitru Mihalache. Models for supercontinuum generation beyond the slowly-varying-envelope approximation. Physical Review A : Atomic, molecular, and optical physics [1990-2015], 2014, 90 (5), pp.053816. 10.1103/PhysRevA.90.053816 . hal-03199192

\section{HAL Id: hal-03199192 \\ https://univ-angers.hal.science/hal-03199192}

Submitted on 15 Apr 2021

HAL is a multi-disciplinary open access archive for the deposit and dissemination of scientific research documents, whether they are published or not. The documents may come from teaching and research institutions in France or abroad, or from public or private research centers.
L'archive ouverte pluridisciplinaire HAL, est destinée au dépôt et à la diffusion de documents scientifiques de niveau recherche, publiés ou non, émanant des établissements d'enseignement et de recherche français ou étrangers, des laboratoires publics ou privés. 


\title{
Models for supercontinuum generation beyond the slowly-varying-envelope approximation
}

\author{
Hervé Leblond, ${ }^{1}$ Philippe Grelu, ${ }^{2}$ and Dumitru Mihalache ${ }^{3,4}$ \\ ${ }^{1}$ LUNAM Université, Université d'Angers, Laboratoire de Photonique d'Angers, EA 4464, 2 Boulevard Lavoisier, \\ 49000 Angers, France \\ ${ }^{2}$ Laboratoire Interdisciplinaire Carnot de Bourgogne, UMR No. 6303 associée au CNRS, Université de Bourgogne, \\ 9 Avenue A. Savary, BP 47870, Dijon Cedex 21078, France \\ ${ }^{3}$ Academy of Romanian Scientists, 54 Splaiul Independentei, 050094 Bucharest, Romania \\ ${ }^{4}$ Horia Hulubei National Institute for Physics and Nuclear Engineering, 30 Reactorului, Magurele, 077125 Bucharest, Romania
}

(Received 1 May 2014; revised manuscript received 15 October 2014; published 10 November 2014)

\begin{abstract}
We show numerically that both the modified Korteweg-de Vries and the sine-Gordon models are conducive to the generation of supercontinua with spectral bandwidths of several octaves, when an intense short pulse is launched as the initial condition. These models beyond the slowly-varying-envelope approximation could play an important role in modeling supercontinuum generation in gas-filled hollow waveguides.
\end{abstract}

DOI: 10.1103/PhysRevA.90.053816

PACS number(s): 42.65.Ky, 42.81.Dp, 42.65.Re

\section{INTRODUCTION}

Supercontinuum generation (SCG) is a matter of extensive research, driven by important applications in spectroscopy, optical coherence tomography, and optical frequency metrology [1]. It has a long-standing research history, starting in 1970 with the first report of a large frequency broadening attributed to self-phase-modulation (SPM) in bulk glass [2]. Before becoming popular with the use of photonic crystal fibers (PCFs) that allowed a drastic reduction of the input seeding pulse energy to the nanojoule level [3], SCG was commonly referred to as the white-light continuum and was typically experimented on in centimeter-thick glasses with ultrashort millijoule seeding pulses [4,5]. A valuable property of supercontinua is their coherence, which was experimentally investigated in [6] and studied theoretically, for the purely temporal (guided) case, in [7]. Supercontinua generally exit their generating media as highly chirped wideband pulses, which find application, for instance, in the seeding of widely tunable parametric amplifiers [8].

Supercontinuum generation in bulk media involves a complex of spatiotemporal mechanisms that includes space-time self-focusing and self-steepening, with the advent of plasma generation that turns the beam collapse into a filamentation process [9]. In the case of SCG in optical fibers, the physical mechanisms involved are purely temporal, which considerably simplifies the modeling of propagation. Yet the complexity inherent to the wide range of frequencies and nonlinear effects involved remains. To give an order of magnitude of the injected pulses intensities that are necessary for SCG, in optical fibers, the pulse peak power typically reaches the $\mathrm{kW}$ level, corresponding to peak intensities of a few $\mathrm{GW} / \mathrm{cm}^{2}[10,11]$. The use of intense femtosecond lasers, such as Ti:sapphire, as seeding sources in microstructured optical fibers allowed the generation of ultrawideband spectra. Around the year 2000, the obtained wideband spectra were spanning over more than one octave, from the visible to the near infrared [3,12]. Optical intensities approaching the damage threshold of silica were also launched in tapered fibers, with the outcome of producing a supercontinuum light extending over more than two octaves, from the ultraviolet to the infrared $(370-1545 \mathrm{~nm}$ at the $-20 \mathrm{~dB}$ level) [13]. Since then, the tapering technology applied to PCF has significantly progressed, allowing one to obtain, for instance, supercontinuum spectra covering a larger domain of the electromagnetic spectrum from ultraviolet to near infrared (350-2400 nm) [14].

The buildup of supercontinuum radiation in fiber waveguides typically involves SPM and Raman scattering nonlinearities. However, the detailed processes at play depend on the duration of the seed pulse and on the properties of the material. For instance, pumping an anomalously dispersive media with pulses of picosecond duration or longer will trigger modulational instability during the initial stage, followed by a breakup into soliton pulses and their collisions in a complex interaction picture including soliton self-frequencyshift and radiation $[15,16]$. In the same type of media, by launching femtosecond pump pulses, a stage of intense spectral broadening will occur, in association with high-order soliton compression, followed by the fission of the high-order soliton. Such fission is highly dependent on higher-order dispersion terms and Raman scattering, which also break the spectral symmetry [17-19].

A widespread propagation model used for SCG is the generalized nonlinear Schrödinger equation, where high-order dispersion terms, self-steepening, and Raman scattering are included [19]. This model assumes a slowly-varying-envelope approximation (SVEA), which is in principle valid for pulses whose duration is several optical cycles long or, equivalently, for an optical bandwidth narrower than the central frequency, even though the validity of carefully derived envelope equations can cover pulse durations down to the single-cycle regime of nonlinear optics [20].

In addition, there exist nonlinear media conducive to supercontinuum generation that do not involve Raman scattering, such as noble gases. The nonlinear efficiency of a noble gas is magnified when it is used as a filler in specially designed hollow-core PCF. Indeed, large spectral broadening extending to the ultraviolet has been observed with an Ar-filled PCF [21]. Raman scattering was absent from that process, which otherwise involves self-phase-modulation as the basis of the spectral broadening effect. Note that the importance 
of a similar spectral broadening was recognized as early as 1976 [22] and it is also involved in high-harmonic generation: It is indeed well known that ultraintense pulses generate high harmonics in noble gases. Whereas at a very high intensity of several tens of $\mathrm{TW} / \mathrm{cm}^{2}$, third and fifth harmonics were generated in air [23], higher-order harmonics were produced in noble gases (Ar and $\mathrm{Kr}$ ) [24].

Envelope models are not adapted to the study of processes involving very broad spectra, spanning over several octaves. Indeed, the SVEA fails completely to be valid, even using correction terms, when the bandwidth is larger than the fundamental frequency. It should be noted that numerical resolution of one-dimensional propagation equations can also be performed using spectral methods, without assuming the SVEA. The validity of such approaches is not questioned here, however the use of those techniques cannot represent a verification of the SVEA approach.

Models beyond the SVEA have been proposed to describe the nonlinear propagation of few-cycle pulses (FCPs) and the formation of FCP solitons. We are mainly interested here in the modified Korteweg-de Vries (mKdV) [25], and sine-Gordon (sG) equations [26], which were initially derived in the frame of a two-level medium, assuming that the resonance frequency was, respectively, either well above or well below the central frequency of the FCP. Assuming the existence of both high- and low-frequency resonances yields the mKdV-sG model [27]. It assumes that the central frequency is far from both low and high transition frequencies. Another non-SVEA model, the so-called short-pulse equation (SPE) [28-31], can be seen as a reduction of the mKdV-sG model [32]. Other models describing few-opticalcycle solitons and pulse compression in nonlinear dispersive media have been also proposed [33-38] (see the reviews in [39-42]).

Our objective is to test fundamental models for supercontinuum generation that would be applicable to simple atomic systems, such as noble gases, under the assumption that the involved optical wavelengths are far from resonance. In such simple systems, all high-order terms, including dispersion ones, are self-contained into a compact field propagation model, which goes beyond the envelope approximation and thus becomes pertinent in few-cycle pulse propagation. We recall that there is a rigorous mathematical procedure to derive the $\mathrm{sG}$ and $\mathrm{mKdV}$ models from the Maxwell-Bloch equations for a two-level system [26], which can be extended to a general Hamiltonian formulation in the latter case [43]. In the present paper we show that both $\mathrm{mKdV}$ and $\mathrm{sG}$ models are able to describe the process of multiple-octavespan supercontinuum generation, with an initial high-intensity ultrashort input pulse. In the following section we derive some useful analytical results from the combined $\mathrm{mKdV}$-sG model. This model is then solved numerically, in Secs. III and IV, in the simpler $\mathrm{mKdV}$ and $\mathrm{sG}$ approximations, respectively. We also briefly analyze the corresponding physical mechanisms behind the subtle process of supercontinuum generation. In Sec. V we analyze the similarities and differences between low-frequency spectra corresponding to both $\mathrm{mKdV}$ and $\mathrm{sG}$ models, at different propagation distances. Section VI summarizes the conclusions of this work.

\section{A FEW ANALYTICAL RESULTS FROM THE mKdV-sG MODEL}

We restrict our study to propagation in transparent media, i.e., in a situation in which absorption and diffusion processes can be neglected, at least in a first level of approximation. Consequently, a natural assumption is that the central frequency of the pulse is far from any resonance frequency of the medium. This assumption is a fortiori required when we consider a very broad spectrum. Recall that, at the considered intensities, resonant linear and overall nonlinear absorption might damage the propagation medium. We assume that the resonance lines of the medium can be separated in two sets: those with frequency well above the central frequency of the pulse and those with frequency well below it. For the former set, the optical pulse can be considered formally as a low-frequency wave; then using the so-called long-wave approximation, the one-dimensional propagation is shown to obey a mKdV-type model $[26,43]$. For the latter set, it is a shortwave approximation that leads to a sG-type model [26,44]. For a more general medium, both types of transitions are present and the propagation of ultrashort pulses can be well described by the mixed mKdV-sG equation [27], which we write in a dimensionless form as

$$
\partial_{z} u+c_{1} \sin \int^{t} u+c_{2} \partial_{t} u^{3}+c_{3} \partial_{t}^{3} u=0
$$

where $u$ is the electric field, $z$ the propagation distance, and $t$ the retarded time, in the reference frame moving at the speed of light in vacuum. Vector forms of Eq. (1) have also been proposed by several authors (see, e.g., Ref. [35]). Since our purpose is not to reproduce a specific experiment but to analyze mechanisms of supercontinuum generation, we shall discuss separately the different contributions to Eq. (1). If $c_{1}=0$, Eq. (1) reduces to the $\mathrm{mKdV}$ equation and accounts for highfrequency transitions only. If $c_{2}=c_{3}=0$, Eq. (1) reduces to the $\mathrm{sG}$ equation and accounts for low-frequency transitions only.

\section{A. Self-phase modulation}

Self-phase modulation is a well-known phenomenon, described in the frame of envelope models and leading to self-broadening of the pulse spectrum. If dispersion can be neglected so that temporal compression does not occur (the so-called thin sample approximation), the SPM propagation problem can be solved analytically (see, e.g., Ref. [45]). We generalize here this solution to the $\mathrm{mKdV}-\mathrm{sG}$ model in order to compare it with the evolution of the spectrum computed numerically.

We assume a quasimonochromatic wave with angular frequency $\omega$ and wave vector $k$, as

$$
u=U(z, t) e^{i(k z-\omega t)}+\text { c.c. }+u_{1}(z, t),
$$

where $U$ is the amplitude of the fundamental wave, c.c. stands for the complex conjugate, and $u_{1}$ is a small correction. Neglecting dispersion, i.e., setting $\partial_{t} u \simeq-i \omega U e^{i(k z-\omega t)}+$ c.c. and so on, assuming a small amplitude so that the sine function can be expanded into a power series of its argument, making 
use of the dispersion relation

$$
k=\frac{-c_{1}}{\omega}-c_{3} \omega^{3},
$$

and disregarding the third-harmonic generation, yields

$$
\partial_{z} U=i\left(\frac{c_{1}}{2 \omega^{3}}+3 \omega c_{2}\right) U|U|^{2} .
$$

Equation (4) is easily solved by separating the amplitude and phase, which yields

$$
U=\frac{A}{2} \exp \left[i\left(\frac{c_{1}}{2 \omega^{3}}+3 \omega c_{2}\right) \frac{A^{2}}{4} z\right] .
$$

Computing numerically the Fourier transform of $U$ as given by Eq. (5) shows the broadening of the spectrum with typical oscillations of the spectral density. These findings will be compared to numerical results below.

\section{B. High-harmonic generation}

Next we consider the phenomenon of harmonic generation as described by the mKdV-sG model. The third-harmonic generation can very easily be evidenced numerically. The correction $u_{1}$ in (2) is written as

$$
u_{1}(z, t)=V(z, t) e^{i\left(k^{\prime} z-3 \omega t\right)}+\text { c.c. }+u_{2}(z, t),
$$

where $V$ is the amplitude of the third harmonic, $k^{\prime}$ its wave vector, and $u_{2}$ a higher-order correction term. The same approximations as above lead to the evolution equation for $V$ as

$$
\partial_{z} V=i\left(\frac{-c_{1}}{6 \omega^{3}}+3 \omega c_{2}\right) U^{3} e^{i\left(3 k-k^{\prime}\right) z}
$$

The solution of Eq. (7), assuming that $U$ is a constant and there is no incident third harmonic $(V=0$ at $z=0)$, leads to well-known oscillations of the third-harmonic amplitude due to the phase mismatch $\Delta k=3 k-k^{\prime}$, with

$$
V \propto \sin \frac{\Delta k z}{2}
$$

It must be noticed that, if SPM is important, the phase of $U$ in Eq. (7) is not constant but evolves according to Eq. (5) and consequently the phase mismatch $\Delta k$ in (8) should be replaced by

$$
\Delta k^{\prime}=3 k-k^{\prime}+3\left(\frac{c_{1}}{2 \omega^{3}}+3 \omega c_{2}\right) .
$$

In numerical computations, the generation of third harmonics as well as higher-order ones is observed. The amplitude of the harmonics oscillates as predicted by (8), but with a different periodicity due to the SPM phenomenon.

An important point for the mechanism of spectral broadening is that the spectrum of the harmonics is wider than the spectrum of the fundamental. Indeed, typically, if $u$ is the fundamental wave, the source for the $n$th harmonic is proportional to $u^{n}$. Assuming that the temporal profile is

$$
u=A e^{-t^{2} / \tau^{2}} e^{-i \omega_{0} t},
$$

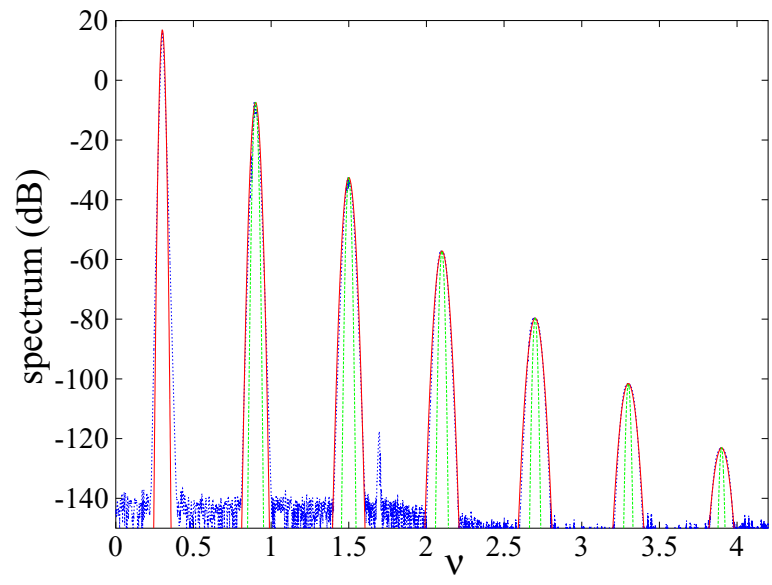

FIG. 1. (Color online) Evolution of the spectral widths of the harmonics with respect to the harmonic order. The blue dotted line is the spectrum of a pulse evolving according to the $\mathrm{mKdV}$ equation at $z=2$, using the initial parameters $\Delta t=100, v=0.3$, and $A=0.7$. The red solid lines are Gaussian fits of the peaks, assuming that the spectral width of the $n$th harmonic is proportional to $\sqrt{n}$, in accordance with analytical results. The green dotted lines are Gaussian spectral profiles having all the same width as the fundamental, for comparison (logarithmic scale).

its Fourier transform, defined by

$$
\hat{u}(\omega)=\frac{1}{2 \pi} \int_{-\infty}^{\infty} u(t) e^{i \omega t} d t,
$$

has the well-known expression

$$
\hat{u}=\frac{1}{A \tau} 2 \sqrt{\pi} e^{-\left(\omega-\omega_{0}\right)^{2} \tau^{2} / 4} .
$$

The $n$th harmonic is then

$$
u^{n}=A e^{-n t^{2} / \tau^{2}} e^{-i n \omega_{0} t}
$$

and its width (the half width at $1 / e$ ) is $\tau / \sqrt{n}$. Consequently, its Fourier transform is

$$
\widehat{\left(u^{n}\right)}=\frac{1}{A \tau} 2 \sqrt{n \pi} e^{-\left(\omega-n \omega_{0}\right)^{2} \tau^{2} / 4 n},
$$

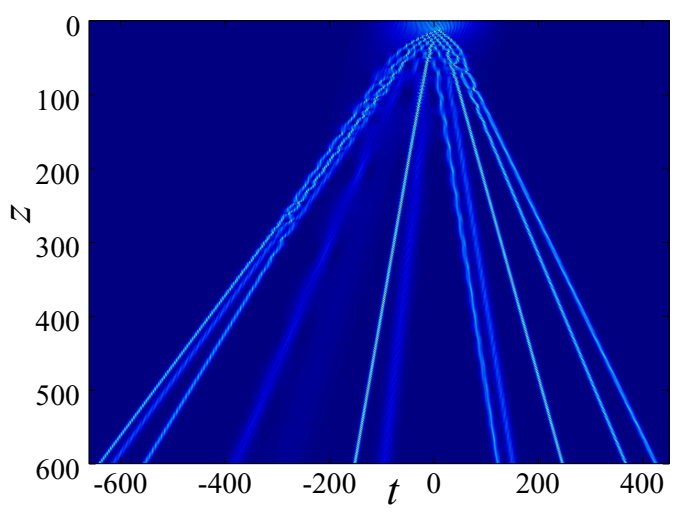

FIG. 2. (Color online) Temporal evolution of an initial pulse with $\Delta t=100, v=0.3$, and $A=0.7$, according to the $\mathrm{mKdV}$ model. The input pulse splits into a set of FCP solitons (the soliton speed is compensated for clarity). 


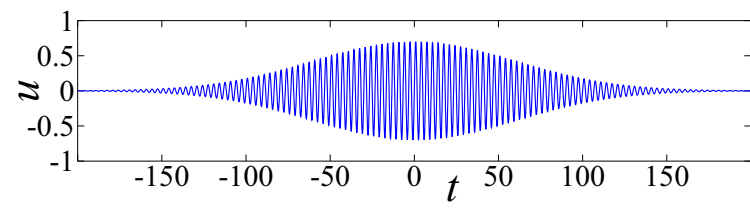

(a)

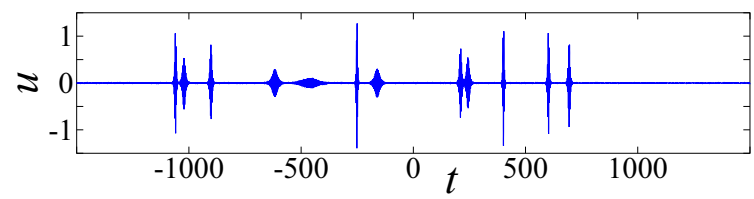

(b)

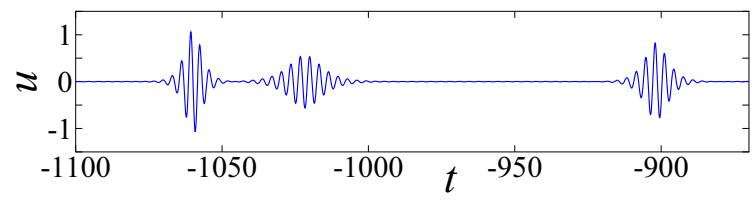

(c)

FIG. 3. (Color online) Evolution of an initial pulse with $\Delta t=$ $100, v=0.3$, and $A=0.7$, according to the mKdV model (same as Fig. 2): (a) initial pulse $z=0$, (b) set of emerging FCP solitons $z=999$, and (c) zoom on some FCP solitons in (b) (the soliton speed is compensated for clarity).

hence the spectral width of the $n$th harmonic is $2 \sqrt{n} / \tau$; it increases as $\sqrt{n}$. This result can be compared with the actual width of the harmonics at the early stage of the pulse evolution. Figure 1 shows a typical pulse spectrum of this kind (namely, the pulse presented in Figs. 2-5, in Sec. III below, for $z=2$ ), with a Gaussian fit of the spectral peaks. The peak height is chosen so that it coincides with numerical results and the solid lines show the dependence of the peak width with respect to the order of harmonics computed above. The spectral width of the fundamental is shown for each peak by the green dotted lines. It can be seen that, despite the spectral profile of the fundamental being closer to a sech-type profile than to a Gaussian one, the evolution of the spectral width with the order

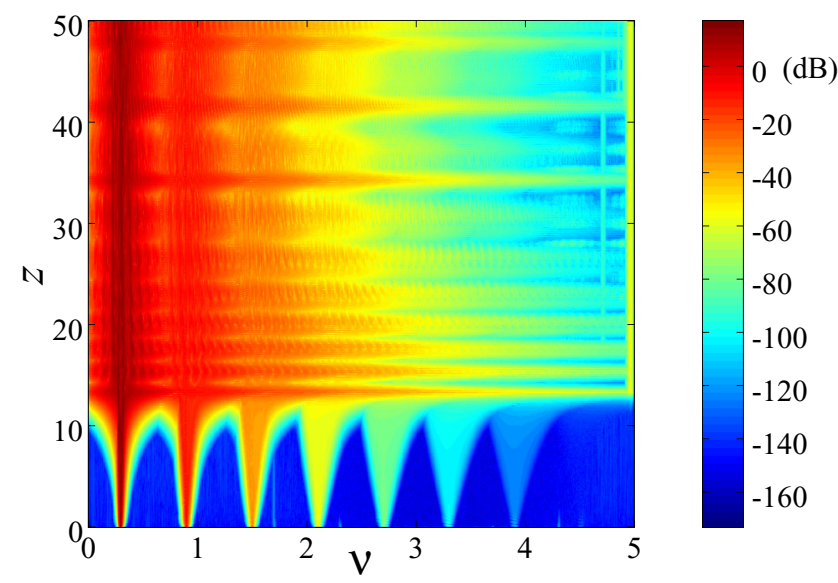

FIG. 4. (Color online) Evolution of the spectrum (on a logarithmic scale) of the initial pulse with $\Delta t=100, v=0.3$, and $A=0.7$, shown in Fig. 2. A very broad spectrum is reached quickly.

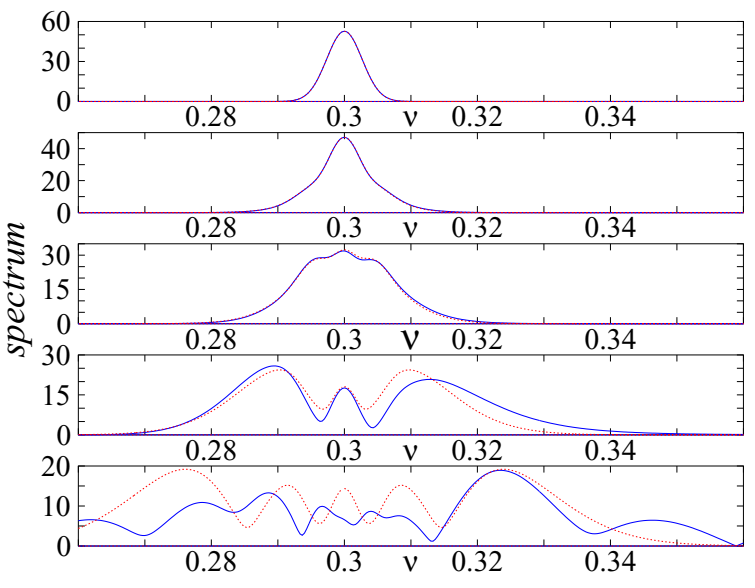

FIG. 5. (Color online) First stage of the evolution according to the mKdV model of the spectrum of the initial pulse with $\Delta t=100$, $v=0.3$, and $A=0.7$, shown in Fig. 2 (blue solid lines), compared to the effect of self-phase modulation (red dotted lines), for $z=0,2$, 4,10 , and 20 .

of the harmonics quite accurately follows Eq. (14). Numerical computation shows that, for the spectrum broadened by the SPM phenomenon, the increase of the spectral width with the order of the harmonic is even larger.

Then it seems that broadening occurs from parametric interaction between the sidebands of the harmonics and the ones of the fundamental. Indeed, if the fundamental wave at frequency $\omega_{0}$ contains a sideband at frequency $\omega_{0}+\delta \omega$, the third harmonics contains the sideband at $3\left(\omega_{0}+\delta \omega\right)$. It may interact with the fundamental at $\pm \omega_{0}$, to yield $3\left(\omega_{0}+\delta \omega\right)-$ $\omega_{0}-\omega_{0}=\omega_{0}+3 \delta \omega$, and other components. The existence of this process has been checked by numerical computation, adding to the initial frequency a small input at a neighbor frequency $\omega_{0}+\delta \omega$; the growth of the frequencies corresponding to the parametric interaction was observed. We suspect that this process is responsible for a part of the spectral broadening.

\section{THE mKdV MODEL}

The mKdV equation, Eq. (1) with $c_{1}=0$, is solved numerically by means of a standard fourth-order Runge-Kutta scheme. The time derivatives are computed using five-point finite differences. We use a large number of discretization points along $t(30000)$ in order to avoid numerical effects due to either high- or low-frequency cutoffs. The use of finite differences rather than a spectral method avoids some drawbacks due to the periodicity in frequency that would be induced by the use of discrete Fourier transforms. However, we use periodic boundary conditions in $t$. The validity of the numerical results was ensured by comparing the results obtained with various step sizes, number of sample points, and numerical schemes, until we obtained results independent of these parameters. In particular, the high-frequency cutoff due to the discretization must be placed above the highest expected harmonics. The initial data has the form of a Gaussian pulse

$$
u(0, t)=A \sin (\omega t) e^{-t^{2} / \tau^{2}},
$$


hence $A$ is the amplitude. For convenience, we use normalized (dimensionless) parameters, assuming $c_{2}=c_{3}=1$ in Eq. (1). We fix the ratio of the (normalized) optical period $2 \pi / \omega$ to the pulse duration $\tau$ to be the same as for a 100 -fs-long pulse with wavelength $\lambda=1 \mu \mathrm{m}$. Therefore, it is more convenient to do as if $t$ was expressed in fs and $\omega=2 \pi c / \lambda$, with $c=0.3 \mu \mathrm{m} / \mathrm{fs}$, and $\tau=\Delta t / \sqrt{2 \ln 2}$, where $\Delta t$ is the full width at half maximum of the pulse. However, since we use normalized quantities, reducing both $\Delta t$ and $\lambda$ (we keep the same ratio throughout the paper) does not correspond really to a reduction of the physical wavelength, but to an increase of the dispersion parameters of the material. The resonance frequencies are supposed, in the frame of the $\mathrm{mKdV}$ model, to be very large and increasing the pulse frequency consists in setting the experimental conditions closer to the resonance.

At a high-amplitude level (computations were run up to $A=2$ ), an initial pulse with $\Delta t=100$ and $v=0.3$ may split into a set of FCP solitons, which are $\mathrm{mKdV}$ breathers (see Figs. 2 and 3). The FCPs form in a first stage until $z \simeq 12$ and then slowly go away one from the other. A huge spectral broadening occurs during the formation of the FCPs, before they begin to separate, as can be seen in Fig. 4 .

Figure 5 presents the first stage of evolution, compared to the effect of SPM as computed from Eq. (5) above. It can be seen that the analytic envelope approximation is quite correct until $z \simeq 2$ and then the actual broadening becomes asymmetric, while the analytic formula remains symmetric. When the supercontinuum is formed, however, the analytically approximated spectrum only accidentally may coincide with the actual one.

At a lower amplitude (about $A=0.1-0.2$ ), the spectrum oscillates, returning to its initial width in a way that recalls the Fermi-Pasta-Ulam recurrence (see Fig. 6). In this case, only two FCP solitons are formed and propagate together as a higher-order soliton of the nonlinear Schrödinger equation does, yielding a kind of superbreather, the evolution of which is shown in Fig. 7.

If we reduce the wavelength $\lambda$ and pulse length $\Delta t$, the dispersion is much higher due to the closeness to resonance.

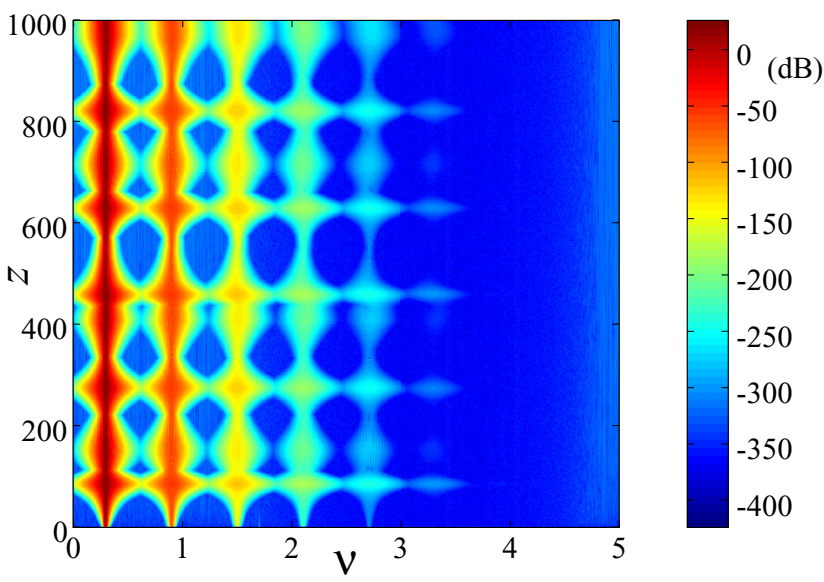

FIG. 6. (Color online) Evolution of the spectrum (on a logarithmic scale) of an initial pulse with $\Delta t=100, v=0.3$, and $A=0.17$. The spectral broadening evolves periodically, showing some recurrence phenomena.

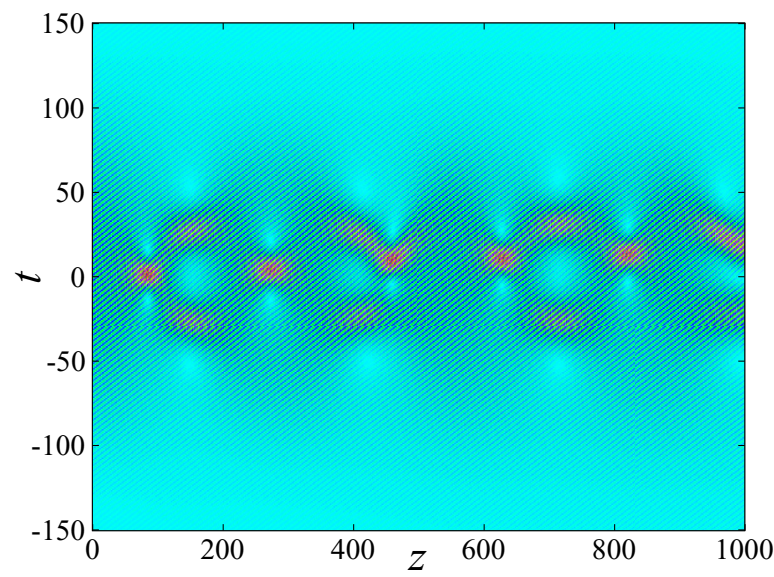

FIG. 7. (Color online) Evolution of the superbreather generated by an initial pulse with $\Delta t=100, v=0.3$, and $A=0.17$.

Consequently, the FCP solitons form at higher amplitude and the evolution from the Gaussian pulse to soliton is much quicker. For $\lambda=0.3$, i.e., $v=1$, and $\Delta t=30$, at $A=0.7$, we observe the same oscillating behavior as for $A=0.17$, $v=0.3$, and $\Delta t=100$ above. For $A=1.5$, still $\Delta t=30$, and $v=1$, the obtained pattern is comparable to Figs. 2 and 3 above, but with six solitons only.

\section{THE sG MODEL}

The sG equation, Eq. (1) with $c_{2}=c_{3}=0$, is solved in the spectral domain by means of a standard fourth-order Runge-Kutta scheme. The antiderivative is computed in the spectral domain, dividing by $i \omega$. The mean value of the function, corresponding to the angular frequency $\omega=0$, is set to zero. Using this method implies that the function $u$ for which the antiderivative is computed should have a zero mean value and that the antiderivative is fixed in such a way that its mean value is zero. The inverse Fourier transform is computed by means of a standard fast Fourier transform algorithm before the sine function is computed, and so on, at each substep of the Runge-Kutta scheme. The number of points is $2^{15}=32768$. We use inputs of the same form (15) as in the previous section, keeping the same ratio $\Delta t / \lambda$, and we set $c_{1}=50$. Note that a change in $c_{1}$ results only in a change of the unit along the $z$ axis. As in the case of the mKdV model, the choice of the numerical scheme and optimum step size was done following a large set of trials, ensuring the accuracy of the numerical solution of the sG equation.

For moderate amplitudes and high frequencies, the SCG process is a slow one. The supercontinuum appears at $z=0.55$ for $\Delta t=100, v=0.3$, and $A=5$ and the distance increases to $z=2.5$ for $\Delta t=80, v=0.375$, and the same amplitude and to $z=3.3$ for $\Delta t=100, v=0.3$, and $A=2.5$. Thus, for moderates amplitudes, e.g., for $A=1$, and for $v=0.3$ and $\Delta t=100$, the supercontinuum appears between $z=9.6$ and 10 and the evolution is much smoother than in the case of high amplitudes. It can be seen that for $A=1$ a few FCP solitons (about six) form and they tend to separate (see Fig. 8).

When the normalized frequency is decreased, still keeping the same ratio between pulse duration $\Delta t$ and wavelength $\lambda$, the 


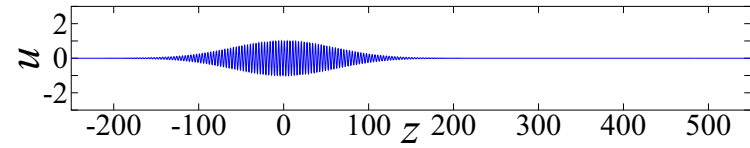

(a)

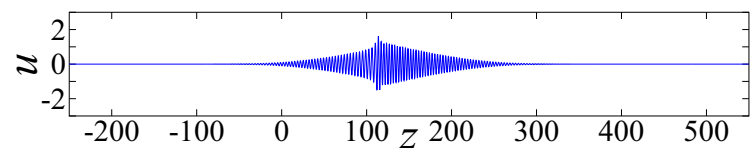

(b)

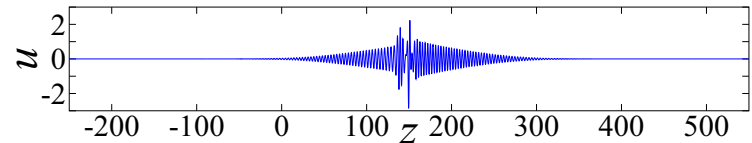

(c)

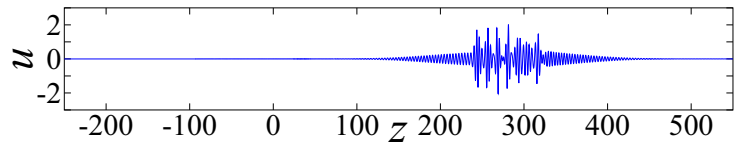

(d)

FIG. 8. (Color online) Evolution of the profile of an initial pulse with $\Delta t=100, v=0.3$, and $A=1$, according to the sG model: (a) input $(z=0)$, (b) formation of the first FCP soliton $(z=9.2)$, (c) the second FCP soliton is just formed $(z=11.16)$, and (d) end of our computation $(z=20)$.

variations represent the dependence with respect to the dipolar momentum of the transition, in other words, the closeness to the resonance, which in this case is assumed to be located at very low frequencies.

For higher amplitude, the nonlinear speed is higher and it is less easily compensated by dispersion. The duration of the FCP solitons tends to decrease and their amplitude to increase. Consequently, their number increases. However, their velocity is smaller and they do not separate. The nonlinear interaction between FCP solitons produces very sharp peaks, which induce numerical instability, so numerical solution cannot be prolonged far away. The nonlinear interaction creates some kind of interference pattern (see Fig. 9). Starting from $z=3.4$, there appear phase dislocations corresponding likely to the

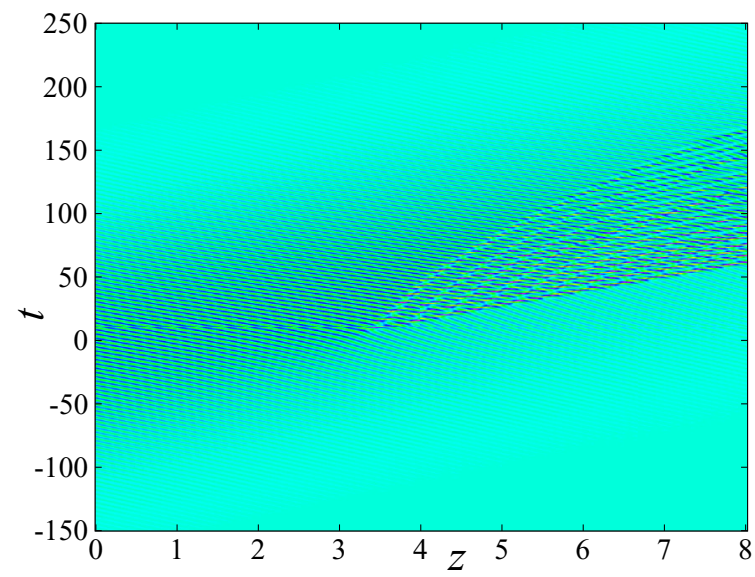

FIG. 9. (Color online) Evolution of an initial pulse with $\Delta t=$ $100, v=0.3$, and $A=2.5$, according to the sG model.

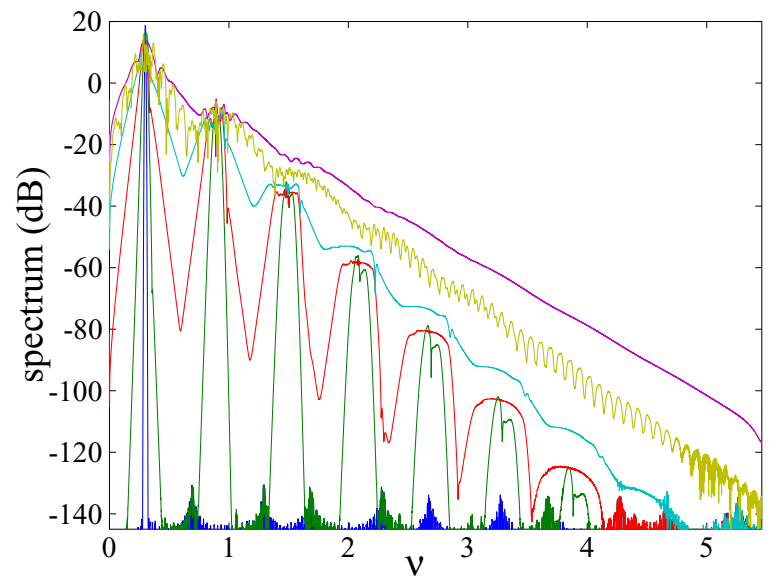

FIG. 10. (Color online) Evolution of the spectrum of an initial pulse with $\Delta t=100, v=0.3$, and $A=1$, according to the sG model (on a logarithmic scale). From bottom to top, $z=0,4,8,9.2$ (arising of the first soliton), 11.16 (the second soliton is just formed), and 20.

formation of FCP solitons and some interference pattern arise between them (see Fig. 9).

At low intensity, only a few harmonics are created (see Fig. 10); however, when the intensity increases, the number of harmonics grows substantially. We note that 15 harmonics are visible in Fig. 11. Oscillations of the amplitude of harmonics can be seen and are more pronounced for higher-order harmonics and for higher intensities. The harmonics are first created and then they broaden until they merge. When they merge, a continuum is formed. At the same time, wave breaking due to nonlinear velocity develops in the time domain. It leads to the formation of a bunch of sharp FCP solitons, when the spectrum becomes very broad.

More specifically, precisely at the propagation distance where the first dislocation of the phase pattern appears in the time domain, a sharp transition occurs in the spectral domain, from a set of broad harmonics to a spectrum monotonically decaying towards high frequencies. The slope of this decay,

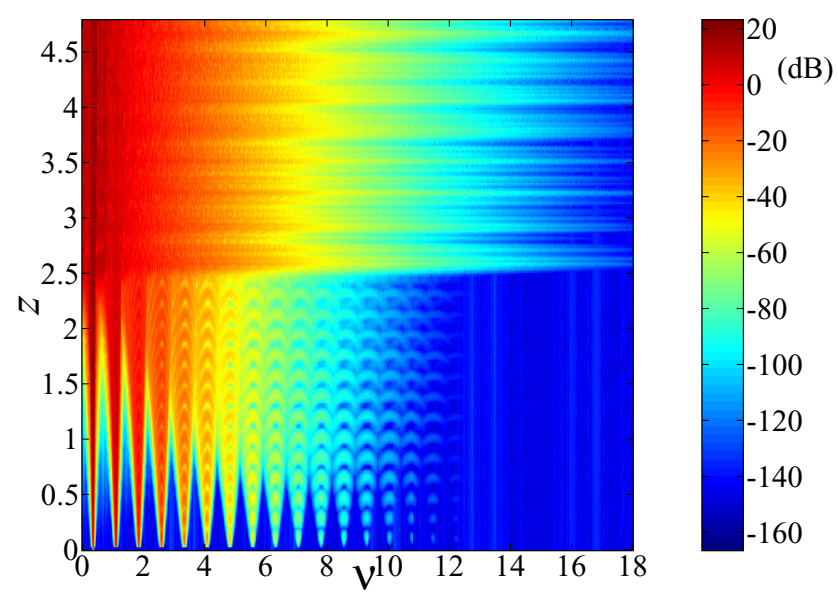

FIG. 11. (Color online) Evolution of the spectrum of an initial pulse with $\Delta t=80, v=0.375$, and $A=5$, according to the $\mathrm{sG}$ model (on a logarithmic scale). Notice the high number of harmonics and the oscillations of their amplitudes. 


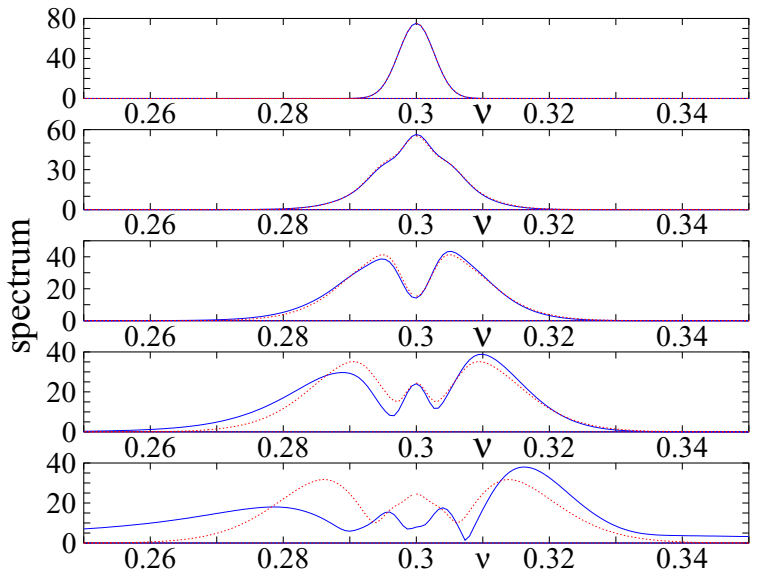

FIG. 12. (Color online) First stage of the evolution of the spectrum of the initial pulse with $\Delta t=100, v=0.3$, and $A=1$, according to the $\mathrm{sG}$ model (blue solid lines), compared to the effect of self-phase modulation (red dotted lines), for $z=0,2.4,4.8,7.2$, and 9.6.

initially identical to the decay of the harmonics, decreases rapidly with further propagation.

Figure 12 is the same as Fig. 5, but for the sG model. If amplitude is increased or frequency is decreased (closer to resonance), the spectrum departs from the precision of the envelope dispersionless computation much faster so that, e.g., in the analogous figure for $\Delta t=80, v=0.375$, and $A=5$, no accordance between both is seen. It should be noted that the asymmetry is inverse to the one observed in the case of the $\mathrm{mKdV}$ model. The numerical curve is above the analytical one for high frequencies, in contrast to what happens for the mKdV model. This is related to the fact that spectral broadening toward low frequencies is more important (see the next section), which reduces the amplitude of the low-frequency sideband of the spectrum in favor of lower frequencies.

\section{THE LOW-FREQUENCY SPECTRUM}

The low-frequency spectrum is more easily seen when the spectrum is plotted against the wavelength $\lambda$. Figure 13 shows this spectrum for both the mKdV model (red dashed line) and the $\mathrm{sG}$ one (blue dash-dotted line). By comparing both curves, we can see that analogous spectrum profiles are obtained. We stress that the distance at which the supercontinuum is produced, the amplitude of the input field, and the strength of nonlinearity and dispersion are intimately related. Consequently, for arbitrary values of the normalized parameters $c_{1}$, $c_{2}$, and $c_{3}$, there is no reason to obtain comparable curves using the same input field amplitudes and the same propagation distances. In Fig. 13, using the scale invariance of both equations, the parameters have been adjusted in order to yield simulations that can be easily compared. More specifically, using the same parameters as above, we obtained comparable spectra for both models with an amplitude $A=0.7$ and a propagation distance $z=16$ for the $\mathrm{mKdV}$ model and $A=2.5$ and $z=4$ for the $\mathrm{sG}$ one. Reducing the parameter $c_{1}$ from 50 to 12.5 changes the latter distance to $z=16$ and reducing $c_{2}$
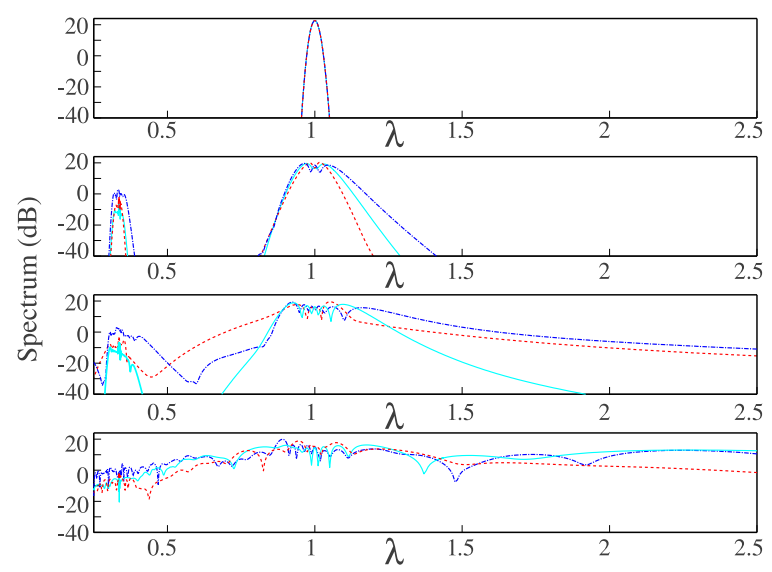

FIG. 13. (Color online) Evolution of the spectrum of an initial pulse with $\Delta t=100, v=0.3$, and $A=2.5$ (on a logarithmic scale, in $\mathrm{dB}$ ), against wavelength $\lambda$, according to three models: the $\mathrm{mKdV}$ one, with $c_{1}=0, c_{2}=0.0784$, and $c_{3}=1$ (red dashed line); the sG one, with $c_{1}=12.5$ and $c_{2}=c_{3}=0$ (blue dash-dotted line); and the $\mathrm{mKdV}$-sG one, with $c_{1}=6.25, c_{2}=0.0392$, and $c_{3}=0.5$ (light blue solid line). The propagation distances are $z=0,6,12$, and 16 .

from 1 to $(0.7 / 2.5)^{2}=0.0784$ changes the former amplitude from 0.7 to 2.5 .

This way the spectra can be compared on a single plot (see Fig. 13). We can notice a few significant differences. For the $\mathrm{mKdV}$ model, the spectrum is always more symmetric than that obtained for the $\mathrm{sG}$ model. The broadening towards high frequencies starts at the current intensity level and decays quite rapidly with decreasing wavelengths, but less rapidly than toward low frequencies. However a wide broadening towards low frequencies also occurs, which decays much slower with wavelength, but forms a kind of pedestal; indeed, it has a maximum intensity much lower than that of the main peak, even at its maximum in $\lambda$. In the range $A \lesssim 0.2$ (for the value $c_{2}=1$ used in Sec. III, which corresponds to $A \lesssim 0.7$ for $c_{2}=0.0784$ ) where superbreathers form and spectral width oscillates, this component of the broadening is much larger with respect to the other one and the asymmetry of the spectrum tends to become in favor of low frequencies.

The final state, when the extremely broad spectrum is formed, is comparable in both cases. However, the first stages of the evolution are quite different. The spectrum in the sG model becomes asymmetric sooner than the $\mathrm{mKdV}$ one and overall the asymmetry arises in the opposite direction. Thus, according to the $\mathrm{mKdV}$ model, the spectrum is more extended toward the short wavelengths first, while according to the sG model, it extends first toward the long wavelengths. This is obviously in relation to the fact that the $\mathrm{mKdV}$ model only accounts for the high-frequency transitions, while the sG model takes into account the low-frequencies transitions only. The Raman effect, due to the interaction of the incident wave with the low-frequency transitions, is known to be an important cause of the self-broadening toward the low frequencies in many experimental situations of SCG. In the present framework, the low frequencies are taken into account by the $\mathrm{sG}$ equation. However, this model is derived by means of 
a short-wave approximation, which formally sets the value of the resonance frequency to zero. Precisely, the sG equation is derived from a two-level Maxwell-Bloch model, which takes the Raman effect into account, by a mathematical procedure that assumes that the Raman shift tends to zero. Hence, the question whether the self-broadening arising from Raman effect is still observed within this approximation naturally arises. Figure 13 shows that a low-frequency spectral broadening qualitatively similar to the action of a Raman effect is actually accounted for by the sG model.

In addition, an example of evolution according to the full mKdV-sG system has been plotted in Fig. 13 (light blue solid line). We choose the parameters providing intermediate values between the two special cases that we just compared: half the value of one of the set of coefficients plus half the value of the other one. This required us to compute the evolution with both the $\mathrm{mKdV}$ and the $\mathrm{sG}$ terms of the equations by using the same numerical scheme. We found that the fourthorder Runge-Kutta scheme in the spectral domain presented for the $\mathrm{sG}$ model could be used, with adequate numerical parameters for the evolution step, spectral resolution, and temporal resolution. In the early stage, the curve is almost exactly in between the other two and the final development of the supercontinuum is also very close to them. However, the intermediate stage of the development occurs slower than in the case of pure $\mathrm{sG}$ or $\mathrm{mKdV}$ models. We have seen that this stage corresponds to the individualization of FCP solitons and that the FCP solitons of the sG model and the ones of the $\mathrm{mKdV}$ model have different properties. It thus can be understood that the cooperation of the two effects is less effective than each one of them acting separately. Finally, we recall that both the $\mathrm{sG}$ and $\mathrm{mKdV}$ models are completely integrable, while the $\mathrm{mKdV}-\mathrm{sG}$ equation for arbitrary parameters is not. This can explain why FCP solitons would form less efficiently in the case of the mixed $\mathrm{mKdV}$-sG model than for the pure $\mathrm{mKdV}$ or $\mathrm{sG}$ ones.

\section{CONCLUSION}

In this work, we have investigated the ability of two generic models beyond the slowly-varying-envelope approximation, namely, the modified Korteweg-de Vries and the sine-Gordon models, to predict the generation of supecontinuum light with a spectral bandwidth extending over a few octaves, when an intense ultrashort pulse is set as the initial condition of the propagation. We have analyzed the similarities and differences between the obtained low-frequency spectra at different propagation distances for both models. Based on our simulations, we have highlighted that a Raman-like effect is acting in the generic sine-Gordon propagation model, as an essential mechanism of the low-frequency spectral broadening.

As a general statement, we believe that the development of universal models for supercontinuum generation, models that do not involve numerous parameters, are helpful in gaining complementary physical insight, even though these models may provide only a qualitative understanding of the underlying dynamics. We point out, however, that specific higher-order-dispersion correction terms can be added into our models to reproduce the particular dispersion properties of an engineered fiber or material. These refinements are left for future investigation. Finally, the results obtained in the present work might motivate further studies in other physical settings involving nonlinear systems, e.g, in the context of hydrodynamic surface gravity waves, where ultrawide spectral broadening has been observed experimentally [46].
[1] Supercontinuum Generation in Optical Fibers, edited by J. M. Dudley and J. R. Taylor (Cambridge University Press, Cambridge, 2010); The Supercontinuum Laser Source: Fundamentals with Updated References, edited by R. R. Alfano (Springer, New York, 2006).

[2] R. R. Alfano and S. L. Shapiro, Phys. Rev. Lett. 24, 592 (1970).

[3] J. K. Ranka, R. S. Windeler, and A. J. Stentz, Opt. Lett. 25, 25 (2000).

[4] R. L. Fork, C. V. Shank, C. Hirlimann, R. Yen, and W. J. Tomlinson, Opt. Lett. 8, 1 (1983).

[5] P. B. Corkum, C. Rolland, and T. Srinivasan-Rao, Phys. Rev. Lett. 57, 2268 (1986).

[6] M. Bellini and T. W. Hänsch, Opt. Lett. 25, 1049 (2000).

[7] J. M. Dudley and S. Coen, Opt. Lett. 27, 1180 (2002).

[8] E. Freysz, J. Plantard, R. Gillet, R. Maleck-Rassoul, Ph. Grelu, and A. Ducasse, Appl. Opt. 37, 2411 (1998).

[9] A. L. Gaeta, Phys. Rev. Lett. 84, 3582 (2000).

[10] M. Andreana, A. Labruyère, A. Tonello, S. Wabnitz, P. Leproux, V. Couderc, C. Duterte, A. Cserteg, A. Bertrand, Y. Hernandez, D. Giannone, S. Hilaire, and G. Huss, Opt. Express 20, 10750 (2012).

[11] S. Coen, A. Hing, L. Chau, R. Leonhardt, J. D. Harvey, J. C. Knight, W. J. Wadsworth, and Ph. St. J. Russell, J. Opt. Soc. Am. B 19, 753 (2002).
[12] J. H. V. Price, W. Belardi, T. M. Monro, A. Malinowski, A. Piper, and D. J. Richardson, Opt. Express 10, 382 (2002).

[13] T. A. Birks, W. J. Wadsworth, and P. St. J. Russell, Opt. Lett. 25, 1415 (2000).

[14] U. Møller, S. T. Sørensen, C. Larsen, P. M. Moselund, C. Jakobsen, J. Johansen, C. L. Thomsen, and O. Bang, Opt. Fiber Technol. 18, 304 (2012).

[15] A. Abeeluck and C. Headley, Opt. Lett. 30, 61 (2005).

[16] S. T. Sørensen, C. Larsen, U. Møller, P. M. Moselund, C. L. Thomsen, and O. Bang, J. Opt. Soc. Am. B 29, 2875 (2012).

[17] A. V. Husakou and J. Herrmann, Phys. Rev. Lett. 87, 203901 (2001).

[18] D. V. Skryabin and A. V. Gorbach, Rev. Mod. Phys. 82, 1287 (2010).

[19] J. M. Dudley, G. Genty, and S. Coen, Rev. Mod. Phys. 78, 1135 (2006); G. Genty, S. Coen, and J. M. Dudley, J. Opt. Soc. Am. B 24, 1771 (2007).

[20] T. Brabec and F. Krausz, Phys. Rev. Lett. 78, 3282 (1997).

[21] N. Y. Joly, J. Nold, W. Chang, P. Hölzer, A. Nazarkin, G. K. L. Wong, F. Biancalana, and P. St. J. Russell, Phys. Rev. Lett. 106, 203901 (2011).

[22] C. Lin and R. H. Stolen, Appl. Phys. Lett. 28, 216 (1976).

[23] G. O. Ariunbold, P. Polynkin, and J. V. Moloney, Opt. Express 20, 1662 (2012). 
[24] S. Hädrich, J. Rothhardt, M. Krebs, F. Tavella, A. Willner, J. Limpert, and A. Tünnermann, Opt. Express 18, 20242 (2010).

[25] I. V. Melnikov, D. Mihalache, F. Moldoveanu, and N.-C. Panoiu, Phys. Rev. A 56, 1569 (1997).

[26] H. Leblond and F. Sanchez, Phys. Rev. A 67, 013804 (2003).

[27] S. V. Sazonov, Zh. Eksp. Teor. Fiz. 119, 419 (2001) [JETP 92, 361 (2001)].

[28] T. Schäfer and C. E. Wayne, Physica D 196, 90 (2004).

[29] A. Sakovich and S. Sakovich, J. Phys. Soc. Jpn. 74, 239 (2005).

[30] Y. Shen, F. Williams, N. Whitaker, P. G. Kevrekidis, A. Saxena, and D. J. Frantzeskakis, Phys. Lett. A 374, 2964 (2010); Y. Shen, N. Whitaker, P. G. Kevrekidis, N. L. Tsitsas, and D. J. Frantzeskakis, Phys. Rev. A 86, 023841 (2012).

[31] E. D. Farnum and J. N. Kutz, J. Opt. Soc. Am. B 30, 2191 (2013).

[32] H. Leblond and D. Mihalache, Phys. Rev. A 79, 063835 (2009).

[33] E. V. Kazantseva, A. I. Maimistov, and B. A. Malomed, Opt. Commun. 188, 195 (2001).

[34] S. V. Sazonov and A. F. Sobolevskii, Zh. Eksp. Teor. Fiz. 123, 1160 (2003) [JETP 96, 1019 (2003)].
[35] S. A. Skobelev, D. V. Kartashov, and A. V. Kim, Phys. Rev. Lett. 99, 203902 (2007).

[36] E. V. Kazantseva, A. I. Maimistov, and J.-G. Caputo, Phys. Rev. E 71, 056622 (2005).

[37] Sh. Amiranashvili, U. Bandelow, and N. Akhmediev, Phys. Rev. A 87, 013805 (2013).

[38] S. V. Sazonov and N. V. Ustinov, Pis'ma Zh. Eksp. Teor. Fiz. 99, 586 (2014) [JETP Lett. 99, 503 (2014)].

[39] S. V. Sazonov, Bull. Russ. Acad. Sci. 75, 157 (2011).

[40] H. Leblond, H. Triki, and D. Mihalache, Rom. Rep. Phys. 65, 925 (2013).

[41] H. Leblond and D. Mihalache, Phys. Rep. 523, 61 (2013).

[42] D. J. Frantzeskakis, H. Leblond, and D. Mihalache, Rom. J. Phys. 59, 767 (2014).

[43] H. Triki, H. Leblond, and D. Mihalache, Opt. Commun. 285, 3179 (2012).

[44] H. Leblond, H. Triki, and D. Mihalache, Phys. Rev. A 86, 063825 (2012).

[45] G. P. Agrawal, Nonlinear Fiber Optics, 5th ed. (Academic, Amsterdam, 2013).

[46] A. Chabchoub, N. Hoffmann, M. Onorato, G. Genty, J. M. Dudley, and N. Akhmediev, Phys. Rev. Lett. 111, 054104 (2013). 